\title{
Dibenzocyclooctadiene lignans from Schisandra spp. selectively inhibit the growth of the intracellular bacteria Chlamydia pneumoniae and Chlamydia trachomatis
}

\author{
Elina Hakala ${ }^{1}$, Leena Hanski ${ }^{1}$, Hanna Uvell ${ }^{2}$, Teijo Yrjönen ${ }^{1}$, Heikki Vuorela ${ }^{1}$, Mikael Elofsson ${ }^{2}$ and \\ Pia Maarit Vuorela ${ }^{1}$
}

\begin{abstract}
Lignans from Schisandra chinensis berries show various pharmacological activities, of which their antioxidative and cytoprotective properties are among the most studied ones. Here, the first report on antibacterial properties of six dibenzocyclooctadiene lignans found in Schisandra spp. is presented. The activity was shown on two related intracellular Gram-negative bacteria Chlamydia pneumoniae and Chlamydia trachomatis upon their infection in human epithelial cells. All six lignans inhibited $C$. pneumoniae inclusion formation and infectious progeny production. Schisandrin B inhibited $C$. pneumoniae inclusion formation even when administered $8 \mathrm{~h}$ post infection, indicating a target that occurs relatively late within the infection cycle. Upon infection, lignan-pretreated $C$. pneumoniae elementary bodies had impaired inclusion formation capacity. The presence and substitution pattern of methylenedioxy, methoxy and hydroxyl groups of the lignans had a profound impact on the antichlamydial activity. In addition our data suggest that the antichlamydial activity is not caused only by the antioxidative properties of the lignans. None of the compounds showed inhibition on seven other bacteria, suggesting a degree of selectivity of the antibacterial effect. Taken together, the data presented support a role of the studied lignans as interesting antichlamydial lead compounds.
\end{abstract}

The Journal of Antibiotics (2015) 68, 609-614; doi:10.1038/ja.2015.48; published online 6 May 2015

\section{INTRODUCTION}

Chlamydia spp. is a genus of Gram-negative intracellular bacteria that are significant agents of human infections. Chlamydia pneumoniae is a frequent cause of community-acquired pneumonia and other respiratory infectious diseases, ${ }^{1}$ and Chlamydia trachomatis, in turn, causes sexually transmitted diseases. C. pneumoniae is the most common chlamydia in humans and nearly everyone is infected 2-3 times in a lifetime. Besides the acute illnesses of respiratory tract, C. pneumoniae has been associated in the pathogenesis of some chronic human diseases. These include chronic obstructive pulmonary disease and asthma, ${ }^{2,3}$ cardiovascular disease, ${ }^{4}$ reactive arthritis ${ }^{5}$ and lung cancer. ${ }^{6}$ C. trachomatis is considered the most prevalent causative agent of sexually transmitted diseases worldwide. ${ }^{7}$ It is also an important causative agent of pelvic inflammatory disease, with complications including infertility ${ }^{8}$. Furthermore, trachoma, a disease caused by ocular C. trachomatis infection, is the leading infectious cause of preventable blindness in the world. ${ }^{9}$

Owing to their obligate intracellular nature Chlamydiae have a characteristic developmental cycle involving different morphological forms of the bacterium. In acute infection the life cycle includes two main forms, infectious extracellular elementary bodies (EBs) and replicating intracellular reticulate bodies. The well-acknowledged problem in the treatment of C. pneumoniae infections is insufficient response to the first line antibiotics, with problems in controlling persistent or relapsing symptoms, or both. ${ }^{10,11}$ In addition to that the infection-induced pathological changes, such as cytokine and chemokine production, cannot be prevented by conventional antibiotics.

The fruits of plant Schisandra chinensis have long been used in traditional Chinese and other oriental medicine. ${ }^{12}$ Recent studies have shown the lignans of $S$. chinensis fruit to possess various pharmacological activities, such as neuro-, cyto- and hepatoprotective, as well as anti-inflammatory effects. ${ }^{13-15}$ The widely studied antioxidant properties of Schisandra lignans are considered one major mechanism mediating the cytoprotective effects. ${ }^{13,16-18}$ Schisandrin B has been shown to inhibit the production of intracellular reactive oxygen species (ROS), which can at least partially be explained by nicotinamide adenine dinucleotide phosphate-oxidase inhibition. ${ }^{19-21}$ C. pneumoniae infection induces ROS production in various cell

\footnotetext{
${ }^{1}$ Pharmaceutical Biology, CDR, Division of Pharmaceutical Biosciences, Faculty of Pharmacy, University of Helsinki, Helsinki, Finland and ${ }^{2}$ Laboratories for Chemical Biology Umeå, Department of Chemistry, Umeå University, Umeå, Sweden

Correspondence: Professor PM Vuorela, Pharmaceutical Biology, CDR, Division of Pharmaceutical Biosciences, Faculty of Pharmacy, University of Helsinki, P.Box 56 (Viikinkaari 5E) FI-00014, Finland.

E-mail: pia.vuorela@helsinki.fi

Received 2 December 2014; revised 1 March 2015; accepted 23 March 2015; published online 6 May 2015
} 
types. ${ }^{22,23}$ C. pneumoniae-infected macrophages use the induction of ROS as a defense response to eliminate the invader. ${ }^{24}$ The ROS in macrophages generated through membrane-associated nicotinamide adenine dinucleotide phosphate-oxidase are known to affect the fate of C. pneumoniae life cycle in these cells. ${ }^{25}$ Regarding the other cell types, it is unclear whether the ROS induction has a role in bacterial survival or reproduction or rather represents an unnecessary or even harmful side effect from the bacterial point of view.

In the current study we report antibacterial activity of six major lignans found in Schisandra spp. with emphasis on their antichlamydial activity.

\section{MATERIALS AND METHODS}

\section{Chemicals}

Schisandrin was purchased from Sigma-Aldrich (St Louis, MO, USA) and schisandrin A, schisandrin B, schisandrin C, schisandrol B and schisantherin A from Fine Tech Industries, London, UK (Table 1). All six lignans were dissolved in dimethyl sulfoxide. L-ascorbic acid, L-dithiothreito and $\mathrm{N}$-acetyl-Lcysteine were dissolved in deionized sterile water.

\section{Cell lines}

Human respiratory tract epithelial HL cells were grown in Roswell Park Memorial Institute medium-1640 medium supplemented with $2 \mathrm{mM}$ L-glutamine, $7.5 \%$ fetal bovine serum and $20 \mu \mathrm{g} \mathrm{ml}^{-1}$ gentamicin, at $37^{\circ} \mathrm{C}, 5 \% \mathrm{CO}_{2}$ and $95 \%$ humidity to confluence in cell culture flasks. ${ }^{26}$ HeLa-229 cells (CCL-2.1; ATCC, Manassas, VA, USA) were propagated in Roswell Park Memorial Institute medium-1640 medium supplemented with $10 \%$ fetal bovine serum, $20 \mathrm{~mm}$ HEPES ( $\mathrm{pH} 8.0$ ), $8 \mu \mathrm{g} \mathrm{ml}^{-1}$ gentamicin, $1 \mu \mathrm{g} \mathrm{ml}^{-1}$ amphotericin $\mathrm{B}$ and $2 \mathrm{~mm}$ L-glutamine at $37^{\circ} \mathrm{C}$, in a humified atmosphere in $5 \% \mathrm{CO}_{2}$. C. pneumoniae clinical isolate $\mathrm{K} 7$ was grown in $\mathrm{HL}$ cells and propagated as described earlier. ${ }^{27}$ C. trachomatis serovar L2 (VR-902B; ATCC) was cultured in HeLa-229 cells and purified as previously described. ${ }^{28}$ Both C. pneumoniae and C. trachomatis were stored in $0.2 \mathrm{M}$ sucrose, $0.02 \mathrm{M}$ sodium phosphate (pH 7.4) and $5 \mathrm{~mm}$ glutamic acid Sucrose-phosphate-glutamic acid (buffer) at $-80^{\circ} \mathrm{C}$ until use.

\section{Infections}

HL cells were seeded into 24 -well plates with coverslips at $4 \times 10^{5}$ cells per well and incubated for $24 \mathrm{~h}$ at $37^{\circ} \mathrm{C}, 5 \% \mathrm{CO}_{2}$ and $95 \%$ humidity. The cell monolayers were inoculated with a suspension of C. pneumoniae EBs diluted in cell culture medium supplemented with $0.5 \mu \mathrm{g} \mathrm{ml}^{-1}$ cycloheximide with the multiplicity of infection 0.2 . The inoculated cells were centrifuged at $550 \mathrm{~g}$ for $1 \mathrm{~h}$ and incubated at $37^{\circ} \mathrm{C}$ for $1 \mathrm{~h}$ to allow the EBs to internalize into the host cells. Medium supplemented with $1 \mu \mathrm{g} \mathrm{ml}^{-1}$ cycloheximide containing the samples or controls at indicated concentrations was added. Non-treated infections, infections treated with $0.01 \mu \mathrm{g} \mathrm{ml}^{-1}$ rifampicin and non-infected cells served as controls. In each well dimethyl sulfoxide was adjusted to $0.25 \%$. Plates were incubated at $37^{\circ} \mathrm{C}, 5 \% \mathrm{CO}_{2}$ and $95 \%$ humidity. Seventy-two hours post infection, the wells were fixed with methanol. The chlamydia inclusions were stained with Pathfinder Chlamydia culture confirmation system (Bio-Rad Laboratories, Berkeley, CA, USA) and inclusion counts were determined under fluorescent microscope with a $\times 200$ magnification.

HeLa-229 cells were seeded in 96-well flat-bottom cell culture plates $\left(1 \times 10^{4}\right.$ cells per well) in Roswell Park Memorial Institute medium-1640 medium supplemented as above. Confluent cells were infected with C. trachomatis serovar L2 diluted in Hank's balanced salt solution at multiplicity of infection 0.3. After $1 \mathrm{~h}$ incubation at $37^{\circ} \mathrm{C}$, Hank's balanced salt solution was replaced with growth medium including test compounds or solvent alone (1\% dimethyl sulfoxide). Cell cultures were fixed at $24 \mathrm{~h}$, immunostained (Pathfinder) and examined at $\times 200$ magnification with fluorescent microscopy.

\section{Evaluation of infectious progeny}

HL cells were seeded into 24-well plates either with or without coverslips, infected and treated with the samples as described above. After $72 \mathrm{~h}$ infection (first passage) the wells with coverslips were fixed and stained to calculate the inclusions. Cell lysates from the wells without coverslips were used to infect fresh HL cell monolayers. After another $72 \mathrm{~h}$ infection (second passage) these samples were fixed and stained to determine the amount of infectious progeny.

\section{Effects on other bacterial species}

Growth inhibition on other bacterial species was investigated by turbidity measurements and resazurin staining. ${ }^{29}$ Gram-negative bacteria; Enterobacter aerogenes, Escherichia coli, Proteus mirabilis, Pseudomonas aeruginosa and Grampositive bacteria; Bacillus subtilis, Staphylococcus aureus and Staphylococcus epidermis (ATCC reference strains) were grown on agar (Bacteriological agar Type A), inoculated to $10 \mathrm{ml}$ of nutrient broth and grown overnight at $37^{\circ} \mathrm{C}$ in a shaker at $100 \mathrm{rpm}$. A $500 \times$ dilution was made in the broth and this suspension was seeded into a 96-well plate. The lignans were added at $50 \mu \mathrm{m}$ concentration and nontreated cultures, cultures treated with either $12 \mathrm{nM}(0.01$ $\mu \mathrm{g} \mathrm{ml}^{-1}$ ) rifampicin or $20 \mu \mathrm{g} \mathrm{ml}^{-1}$ ampicillin as well as only nutrient broth served as control samples. Starting point turbidity was measured as light absorbance at $590 \mathrm{~nm}$ with VarioSkan Flash plate reader (Thermo Fischer Scientific, Vantaa, Finland). The plates were incubated for $24 \mathrm{~h}$ on a shaker at $37^{\circ} \mathrm{C}, 100 \mathrm{rpm}$. The change in turbidity was measured at $590 \mathrm{~nm}$. Resazurin at $20 \mu \mathrm{M}$ in phosphate-buffered saline was added and incubated for $5 \mathrm{~min}$ at $37^{\circ} \mathrm{C}$, on a shaker in darkness and the fluorescence was measured with VarioSkan flash plate reader at 560/590 $\mathrm{nm}(\mathrm{ex} / \mathrm{em})$.

Table 1 Names and chemical structures of the assayed schisandra lignans

\begin{tabular}{|c|c|c|c|c|c|c|c|c|}
\hline Schisandra lignan & $R_{1}$ & $R_{2}$ & $R_{3}$ & $R_{4}$ & $R_{5}$ & $R_{6}$ & $R_{7}$ & Synonyms ${ }^{\mathrm{a}}$ \\
\hline Schisandrin & $\mathrm{OCH}_{3}$ & $\mathrm{OCH}_{3}$ & $\mathrm{OCH}_{3}$ & $\mathrm{OCH}_{3}$ & $\mathrm{H}$ & $\mathrm{OH}$ & $\mathrm{CH}_{3}$ & Schizandrin, Schisandrol A, wuweizi alcohol A, wuweizichun A \\
\hline Schisandrin A & $\mathrm{OCH}_{3}$ & $\mathrm{OCH}_{3}$ & $\mathrm{OCH}_{3}$ & $\mathrm{OCH}_{3}$ & $\mathrm{H}$ & $\mathrm{H}$ & $\mathrm{CH}_{3}$ & Deoxyschisandrin, dezoxischizandrin, wuweizisu $\mathrm{A}$, dimethylgomisin $\mathrm{J}$ \\
\hline Schisandrin B & \multicolumn{2}{|c|}{$-\mathrm{O}-\mathrm{CH}_{2}-\mathrm{O}$} & $\mathrm{OCH}_{3}$ & $\mathrm{OCH}_{3}$ & $\mathrm{H}$ & $\mathrm{H}$ & $\mathrm{CH}_{3}$ & $( \pm$ )- $\gamma$-schizandrin, deoxygomisin A, wuweizisu B \\
\hline Schisandrin C & \multicolumn{2}{|c|}{$-\mathrm{O}-\mathrm{CH}_{2}-\mathrm{O}$} & \multicolumn{2}{|c|}{$-\mathrm{O}-\mathrm{CH}_{2}-\mathrm{O}$} & $\mathrm{H}$ & $\mathrm{H}$ & $\mathrm{CH}_{3}$ & Wuweizisu C \\
\hline Schisandrol B & \multicolumn{2}{|c|}{$-\mathrm{O}-\mathrm{CH}_{2}-\mathrm{O}$} & $\mathrm{OCH}_{3}$ & $\mathrm{OCH}_{3}$ & $\mathrm{H}$ & $\mathrm{OH}$ & $\mathrm{CH}_{3}$ & Gomisin A, wuweizi alcohol B, wuweizichun B, TJN 101 \\
\hline Schisantherin A & \multicolumn{2}{|c|}{$-\mathrm{O}-\mathrm{CH}_{2}-\mathrm{O}$} & $\mathrm{OCH}_{3}$ & $\mathrm{OCH}_{3}$ & $\mathrm{O}-\mathrm{C}-\mathrm{O}-\mathrm{Bz}$ & $\mathrm{CH}_{3}$ & $\mathrm{OH}$ & Gomisin C, schizandrer A, wuweizi ester A \\
\hline
\end{tabular}

asynonyms, Opletal et al. ${ }^{17}$ 


\section{Intracellular ROS detection}

To determine changes in intracellular ROS levels, HL cells were seeded into 96-well plates to 60000 cells per well, incubated overnight and loaded with $20 \mu \mathrm{M}$ 2'7'-dichlorodihydrofluorescein diacetate (DCFH-DA) for $30 \mathrm{~min}$ at $37^{\circ} \mathrm{C}$. The solution was removed from the wells and washed once with Hank's balanced salt solution prior to the exposure of the cells to the lignans for $1 \mathrm{~h}$ at $37^{\circ} \mathrm{C}$. The fluorescence (relative fluorescence units) was measured at 503/523 (ex/em) with VarioskanFlash plate reader. $1 \mathrm{~mm}$ hydrogen peroxide and $\mathrm{N}$-acetylcysteine were used as positive and negative controls. To determine the possible direct interaction of the lignans with the dye, a similar experiment was conducted with no cells (compounds and DCFH-DA). The potential interference on dye de-esterification or efflux was excluded with a 2'7'-dichlorofluorescein diacetate (DCF-DA) assay.

\section{Host cell viability assay}

Effect of the lignans on host cell viability was determined by a resazurin assay as recently described ${ }^{30}$ in two phases of cell growth. The effect on logarithmically dividing cells and confluent cell cultures were assayed with a density of $5 \times 10^{4}$ and $6 \times 10^{5}$ cells per well, respectively. In brief, HL cells seeded into a 96-well plate were incubated overnight and exposed to the test compounds for $72 \mathrm{~h}$ at $37^{\circ} \mathrm{C}$. Viability was detected with a profluorescent probe, resazurin and the fluorescence was measured with VarioSkan Flash plate reader at 570/590 nm (ex/em). Concentration of dimethyl sulfoxide in the sample wells was $0.25 \%$. Triton $\mathrm{X}$ was used as a positive control.

\section{Data analysis}

The average inclusion counts were determined by counting altogether 12 eye fields for each sample (four eye fields per coverslip from experiments with three coverslips). The reductions in inhibition counts were calculated by comparing the average inclusion counts in a treated sample to the average inclusion counts in the untreated infection controls. Data were expressed as means \pm s.e.m., and

Table 2 MIC values of Schisandra lignans against $C$. pneumoniae and C. trachomatis

Schisandra lignan $\quad M I C(\mu M)$ on $\mathrm{C}$. pneumoniae $\quad M I C(\mu \mathrm{M})$ on $\mathrm{C}$. trachomatis

\begin{tabular}{lrc}
\hline Schisandrin & 100 & - \\
Schisandrin A & 50 & 100 \\
Schisandrin B & 50 & 100 \\
Schisandrin C & 25 & 50 \\
Schisandrol B & 100 & - \\
Schisantherin A & 50 & 100
\end{tabular}

Human epithelial HL cells were infected with C. pneumoniae strain $\mathrm{K} 7$ and the effect of the lignans on $C$. pneumoniae inclusion counts after the $72 \mathrm{~h}$ infection was determined using immunofluorescence with a fluorescein isothiocyanate-conjugated genus-specific anti-LPS antibody $C$. trachomatis inclusion count in HeLa cells was determined after $24 \mathrm{~h}$ infection. To determine the MIC-value the lignans were assayed using seven different concentrations: 1.0 determine the Mic-value the lignans were assayed using seven differe statistical analyses were performed by the Student's $t$-test using SPSS Statistics 21.0 software. $P$-values below 0.05 were considered statistically significant.

\section{RESULTS}

Chlamydia growth inhibition and host cell viability

Schisandrin B was identified as a hit compound in our recent highcontent screening study (Hanski et al., unpublished data) using a novel screening platform to identify antichlamydial compounds. ${ }^{31}$ Based on these findings, six dibenzocyclooctadiene lignans originating from Schisandra spp. (Table 1) were assayed against C. pneumoniae and C. trachomatis at varying concentrations. MIC values of the lignans ranged from $>100$ to $25 \mu \mathrm{m}$ when assayed against C. pneumoniae, and from no inhibition to $50 \mu \mathrm{M}$ when assayed against $C$. trachomatis (Table 2). All of the lignans showed inhibition in a dose-dependent manner (Figure 1a). The estimated $\mathrm{IC}_{50^{-}}$values for the lignans against C. pneumoniae were 52, 10, 5, 5, 6 and $20 \mu \mathrm{M}$ for schisandrin, schisandrin A, schisandrin B, schisandrin C, schisandrol B and schisantherin $\mathrm{A}$, respectively.

The effect of the lignans on the host cell viabilities assayed on confluent cells (60 000 cells per well) ranged from 90.3 to $122.0 \%$ The corresponding numbers when assayed with growing cells (5000 cells per well) were from 72.7 to $97.4 \%$. Lignans did not significantly reduce the viability of either confluent or growing cells except schisandrin, which reduced the viability of the growing cells by 18.9\% $(P<0.01)$.

\section{Impact of administration time on inhibitory activity}

Schisandrin and schisandrin B were administered to C. pneumoniaeinfected cell cultures 2, 8 and $24 \mathrm{~h}$ after the bacterial inoculation at a concentration of $50 \mu \mathrm{M}$ (Figure 1b). Delaying the administration of schisandrin $\mathrm{B}$ and schisandrin to $8 \mathrm{~h}$ did not affect their ability to inhibit C. pneumoniae inclusion formation, and partial inhibitory activity remained also upon administration of the lignans at $24 \mathrm{~h}$ post infection.

\section{Inhibition of infectious progeny}

The six lignans were assayed for their effect on $C$. pneumoniae infectious progeny production at 25 and $50 \mu \mathrm{M}$ concentrations (Figure 2). Consistent with previous experiments above, the inhibition of inclusion formation at the first passage at 25 and $50 \mu \mathrm{m}$ were lowest at $8.1 \%$ and $26.3 \%$, respectively with schisandrin. Inhibition percentages were highest with schisandrin $\mathrm{C}(99.5 \%, 25 \mu \mathrm{M})$, schisandrin B, schisandrin C and schisantherin A $(100.0 \%, 50 \mu \mathrm{M})$, each. Regarding the progeny production (inclusion counts in the passaged samples), the inhibition percentages at 25 and $50 \mu \mathrm{m}$ were lowest with
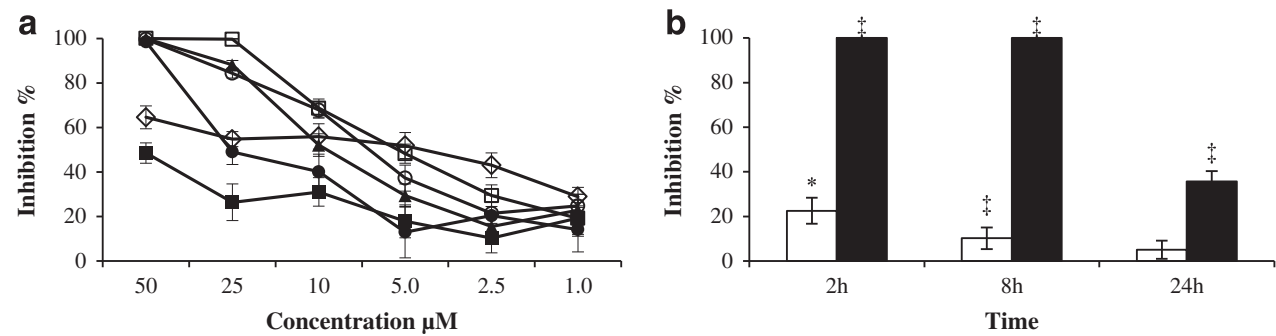

Figure $1 \mathrm{~A}$ Suppression of $C$. pneumoniae inclusion counts by the Schisandra lignans. The growth inhibition of $C$. pneumoniae is expressed as \% using the infected nontreated control as $0 \%$ and the uninfected control as $100 \%$ The data represent mean \pm s.e.m., $n=12$. $\boldsymbol{\Delta}=$ schisandrin; $\boldsymbol{\square}=$ schisandrin $\mathrm{A}$; $\checkmark=$ schisandrin $\mathrm{B} ; \circ=$ schisandrin $\mathrm{C} ; \diamond=$ schisandrol B; $\square=$ schisantherin A. (b) The impact of delayed administration of schisandrin (white bars) and schisandrin B (black bars) on C. pneumoniae inclusion counts. The lignans were administered at $50 \mu \mathrm{m}$ concentration at either $2 \mathrm{~h}, 8 \mathrm{~h}$ or $24 \mathrm{~h}$ post infection. Data represent mean \pm s.e.m., $n=12$. $\left({ }^{*} P<0.05, \dagger P<0.01, \ddagger P<0.001\right)$. 


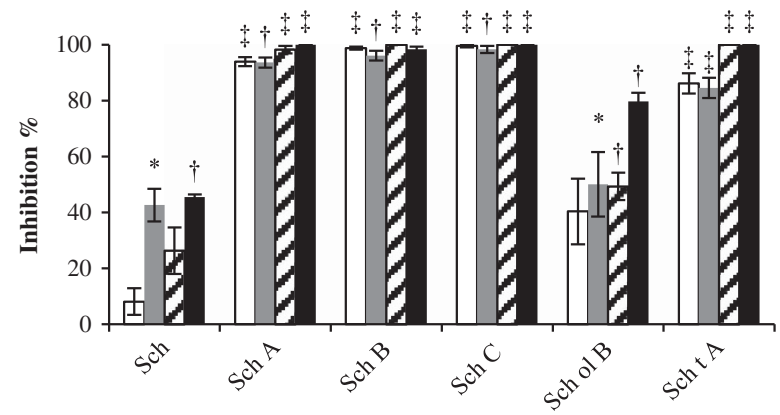

Figure 2 Effect of the Schisandra lignans on C. pneumoniae infectious progeny production. The inclusion counts were determined at the first and the second passage of the infection (see section 2.4.). The lignans were assayed at concentrations of 25 and $50 \mu \mathrm{m}$. Sch=schisandrin, Sch $A=$ schisandrin $A$, Sch $B=$ schisandrin $B$, Sch $C=$ schisandrin $C$, Sch ol $B=$ schisandrol $B$, Sch $t A=$ schisantherin $A$. First passage $25 \mu \mathrm{m}$ : white bars, second passage $25 \mu \mathrm{m}$ : gray bars, first passage $50 \mu \mathrm{m}$ : bars with downward diagonal lines, second passage $50 \mu \mathrm{m}$ : black bars. Data represent mean \pm s.e.m., $n=4$. $\left({ }^{*} P<0.05, \dagger P<0.01, \ddagger P<0.001\right)$.

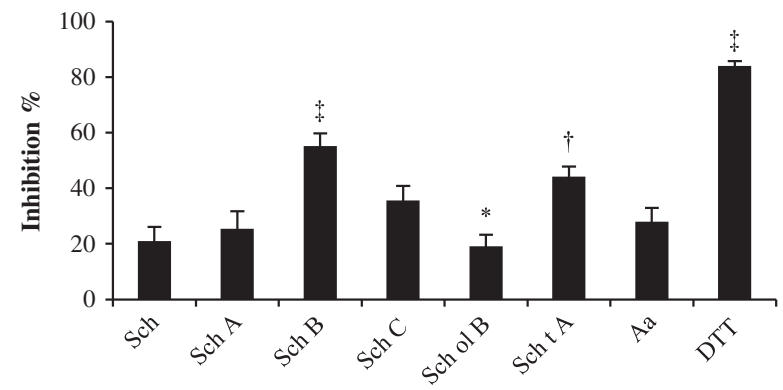

Figure 3 To evaluate direct effects of the lignans on chlamydial elementary bodies, $C$. pneumoniae stocks were diluted in the infection medium to yield a $400000 \mathrm{IFU} \mathrm{ml} \mathrm{m}^{-1}$ suspension. The elementary bodies in the suspension were pretreated at $+4{ }^{\circ} \mathrm{C}$ with $50 \mu \mathrm{m}$ lignans for $1 \mathrm{~h}$ prior to the inoculation on $\mathrm{HL}$ cell monolayers. Sch $=$ schisandrin, Sch $A=$ schisandrin $A$, Sch $B=$ schisandrin $B$, Sch $C=$ schisandrin $C$, Sch ol $B=$ schisandrol $B$ Sch $t$ $A=$ schisantherin $A, A a=$ ascorbic acid $250 \mu \mathrm{m}, D T T=10 \mathrm{~mm}$ dithiotreitol. Data represent mean \pm s.e.m., $n=12 .\left({ }^{*} P<0.05, \dagger P<0.01, \ddagger P<0.001\right)$.

schisandrin, $42.6 \%$ and $45.6 \%$, respectively, and highest with schisandrin C, 98.3\%, and schisandrin A, schisandrin C and schisantherin A $100.0 \%$, respectively. The highest inhibition percentages were reached with schisandrin $\mathrm{C}$ at both concentrations and both passages.

\section{Inhibition of EB-infectivity}

To study the impact of the lignans on C. pneumoniae extracellular form and its entry to the host cell, a pretreatment of $C$. pneumoniae EBs was carried out. When the chlamydial EB particles were pretreated with the lignans at $50 \mu \mathrm{M}$ a partial inhibition of inclusion formation was observed (Figure 3 ). The inhibition percentages were lowest with schisandrin, $11.2 \%$ and highest with schisandrin $\mathrm{B}, 50.2 \%$.

\section{Effects on other bacteria}

To evaluate the sensitivity of bacteria, other than Chlamydiae, to the lignans, the compounds' inhibition of the bacteria was assayed at $50 \mu \mathrm{M}$ of the lignan against both Gram-negative ( $P$. aeruginosa, E. aerogenes, P. mirabilis, E. coli) and Gram-positive (S. epidermis,

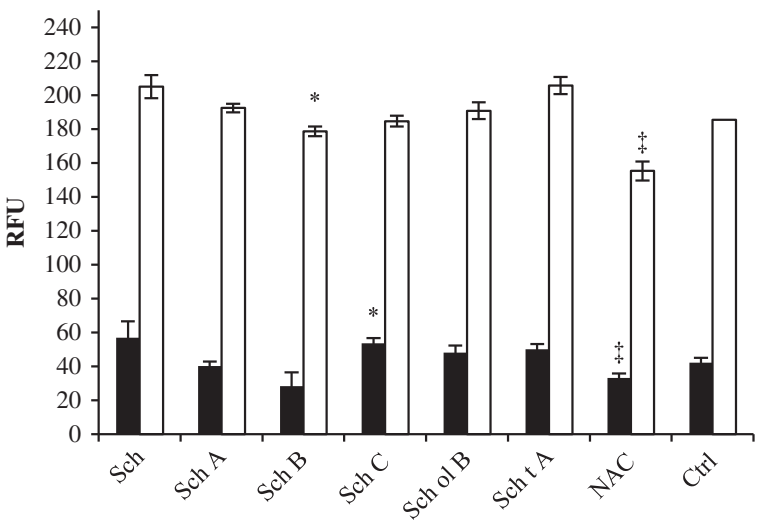

Figure 4 The impact of the Schisandra lignans on basal and infectionassociated intracellular ROS levels. For basal experiments (black bars): HL cells were loaded with the ROS probe DCFH-DA for $1 \mathrm{~h}$, after which the cells were incubated with $50 \mu \mathrm{m}$ lignans for an additional hour before the fluorescent readout. For infection-associated experiments (white bars): HL cells were loaded with DCFH-DA and infected with $C$. pneumoniae MOI 1 simultaneously with $50 \mu \mathrm{m}$ lignans. Intracellular ROS levels were measured after $2 \mathrm{~h}$. Sch $=$ schisandrin, Sch $A=$ schisandrin $A$, Sch $B=$ schisandrin $B$, Sch $C=$ schisandrin $C$, Sch ol $B=$ schisandrol $B$, Sch $t A=$ schisantherin $A$. $10 \mathrm{~mm} N$-acetylcystein (NAC) was used as a positive control. Data represent mean \pm s.e.m., $n=4$. $\left({ }^{*} P<0.05, \dagger P<0.01, \ddagger P<0.001\right)$.

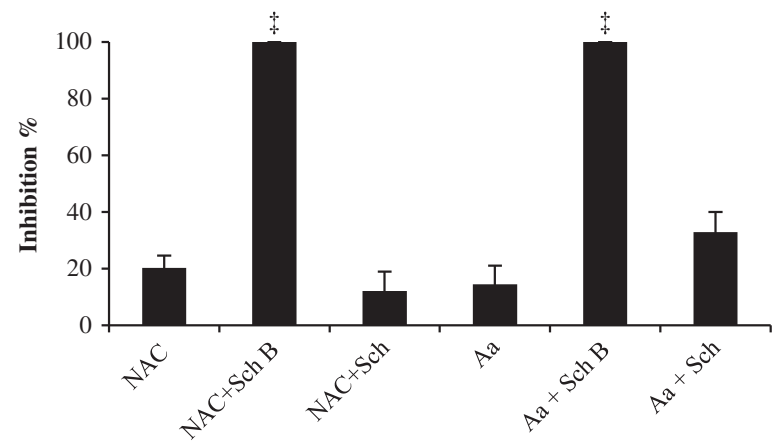

Figure 5 Effect of ascorbic acid and $\mathrm{N}$-acetylcysteine on $\mathrm{C}$. pneumoniae inclusion counts alone and in combination with schisandrin and schisandrin B. C. pneumoniae infections were treated with either $250 \mu \mathrm{g} \mathrm{ml} \mathrm{l}^{-1}$ ascorbic acid (Aa), $10 \mathrm{~mm} \mathrm{~N}$-acetylcysteine (NAC) or Aa in combination with $50 \mu \mathrm{m}$ lignans. Data represent mean \pm s.e.m., $n=12 . \quad\left({ }^{*} P<0.05, \quad \dagger P<0.01\right.$, $\ddagger P<0.001)$.

S. aureus, B. subtilis) bacteria. The investigated lignans did not show growth inhibition of these bacteria (Supplementary Information S1).

\section{Intracellular ROS levels}

To evaluate the impact of the lignans on cellular basal ROS levels, HL cells preloaded with the ROS probe DCFH-DA were exposed to each of the lignans. In these experiments, $25 \mu \mathrm{M}$ schisandrin $\mathrm{A}$ and schisandrin B reduced the amount of intracellular ROS with $30.5 \%$ and $32.3 \%$, respectively $(P<0.01)$. At $50 \mu \mathrm{m}$ only schisandrin $\mathrm{C}$ caused slight change in ROS level (Figure 4, black bars). When $1 \mathrm{mM} \mathrm{H}_{2} \mathrm{O}_{2}$ was applied to induce an increase in intracellular ROS levels, none of the lignans at 25 or $50 \mu \mathrm{M}$ either increased or decreased the amount of ROS (data not shown). In C. pneumoniae-infected cells (multiplicity of infection 1.0), only $50 \mu \mathrm{m}$ schisandrin B reduced the amount of ROS by $6.5 \%(P<0.05$, Figure 4 , white bars). The amount of ROS as 
such was not significantly increased upon C. pneumoniae infection compared with uninfected controls.

None of the lignans at 25 or $50 \mu \mathrm{m}$ concentration either increased or decreased the amount of fluorescence produced by the DCFH-DA probe in a control experiment conducted without HL cells (data not shown). When incubated with the oxidized form of probe (DCFHDA), only schisandrin B at $25 \mu \mathrm{M}$ had a diminishing effect on the fluorescent signal $(37.6 \%$ of control, $P<0.05)$, indicating that the observed effects may partially result from altered probe distribution or deacetylation by the sample. The effect was not observed at $50 \mu \mathrm{M}$.

\section{The effect of other antioxidants on C. pneumoniae inclusion formation}

To evaluate the ability of other known antioxidants to inhibit C. pneumoniae inclusion formation and affect the inhibitory activity of Schisandra lignans, ascorbic acid and $\mathrm{N}$-acetylcysteine were assayed alone and in combination with schisandrin and schisandrin B. Ascorbic acid and $\mathrm{N}$-acetylcysteine yielded a small, statistically non-significant effect on $C$. pneumoniae inclusion formation at concentrations generally known effective in ROS scavenging and diminishing oxidative stress (Figure 5). Furthermore, neither ascorbic acid nor $\mathrm{N}$-acetylcysteine affected the inhibitory activity of schisandrin or schisandrin B when they were administered concomitantly to the infected HL cell cultures.

\section{DISCUSSION}

Although widely studied, there are no previous reports considering Schisandra lignans' antibacterial effects. In the current work, we report that the dibenzocyclooctadiene lignans from Schisandra spp. are potent chlamydia inhibitors with dose-dependent effects on the growth of Chlamydia in vitro. The inhibition was established with two different chlamydial strains, C. pneumoniae clinical pneumonia isolate $\mathrm{K} 7$ and C. trachomatis serovar L2, a lymphogranuloma venereum strain with invasive properties. Complete eradication of chlamydial inclusions was achieved on both species but the lignans did not have an inhibitory effect on seven other studied bacteria, indicating a degree of selectivity for the antichlamydial effect. Besides reducing chlamydial inclusion counts in infected cell cultures, all six lignans inhibited formation of infectious progeny at concentrations of 25 and $50 \mu \mathrm{M}$ (Figure 2). This indicates that the inhibitory activity of these compounds is not likely to involve any drastic effects on events occurring late during the replication cycle, such as reticulate body to EB maturation or exit of EBs from the host cell.

In delayed administration time experiments, schisandrin and schisandrin B maintained their inhibition on C. pneumoniae growth when they were added into the infected cell cultures $8 \mathrm{~h}$ after the initiation of the infection and schisandrin B partially inhibited the inclusion formation even when added at $24 \mathrm{~h}$ post infection (Figure 1b). These results indicate that the observed inhibitory effects are not caused by events related to $C$. pneumoniae entry into the host cell or early stages of its replication cycle, such as establishment of inclusions or EB to reticulate bodies differentiation.

The small differences in the chemical structures of the studied lignans resulted in differential antichlamydial activities (Table 2). The most active lignin schisandrin $\mathrm{C}$ harbors a methylenedioxy bridge at R3 and R4, whereas the schisandrin B has methoxy groups at R3 and R4. A pairwise comparison of schisandrin and schisandrol B, as well as schisandrin A and schisandrin B revealed a similar structure-activity pattern. Also earlier studies suggest the methylenedioxy group to be a structural determinant of biological activity of these lignans. ${ }^{32,33}$ Another structural determinant identified in this study was that schisandrin and schisandrin A differ from each other only by a hydroxyl group at R6 (present in schisandrin, missing in schisandrin A), schisandrin A being significantly more active than schisandrin. A similar pattern is seen also between schisandrol B and schisandrin B. Consistent with various earlier studies comparing these compounds ${ }^{13,17,18,34}$ schisandrin B appears to bear higher biological activity than schisandrin.

We also examined whether the lignans have a direct inhibitory effect on the chlamydial EB infectivity. Pretreatment of C. pneumoniae EBs with the Schisandra lignans resulted in inhibition of chlamydial inclusion formation in the following infection (Figure 3). These effects may not be directly associated with the observed growth inhibition when treating the cellular infection (described above). As the inhibitory effect is maintained when the compound is administered $8 \mathrm{~h}$ post infection, a time point reflecting a stage in which majority of EBs have differentiated into reticulate bodies, ${ }^{35}$ these effects cannot be explained by the potential interference of $\mathrm{EB}$ integrity by the compounds.

As Schisandra lignans have been widely studied for their antioxidant and pro-oxidant properties, ${ }^{18,22,36,37}$ and $C$. pneumoniae infection is known to induce an increase in host cell ROS levels, ${ }^{22,23}$ the relevance of this aspect in the antichlamydial effect of the lignans was studied. However, the results indicated that the antichlamydial activity and effects on cellular ROS levels were not directly correlated and coadministration of the lignans with ascorbic acid or $\mathrm{N}$-acetylcysteine did not affect their antichlamydial activity. Also the time course of C. pneumoniae-induced increase in ROS levels and that of the antichlamydial effect of schisandrin B does not support the role of ROS in the mechanism of the inhibitory effect. C. pneumoniaeinduced ROS production has been reported to occur shortly after the entry into host cell, typically within $1 \mathrm{~h}$ after the initial contact between the bacterium and the host cell, whereas the results from the experiment with delayed administration of schisandrin B demonstrate an efficient inhibition even at $8 \mathrm{~h}$ administration (Figure $1 \mathrm{~b}$ ).

Toxicology of Schisandra lignans and extracts has been studied in vitro and in vivo and they are generally considered nontoxic. ${ }^{15,22,36,38,39}$ Consistent with the literature, in this study the lignans did not affect the host cell viabilities at the studied concentrations. Besides safety, gastrointestinal absorption is a key requirement for a successful lead compound. Earlier studies have demonstrated in vivo bioactivities of oral doses of schisandrin B or $\mathrm{C},{ }^{32,40}$ thus indicating the bioavailability of these compounds when orally administered.

Taken together, these data suggest that the antichlamydial activities of Schisandra lignans are targeted to the mid-phase of the intracellular replication cycle and are not solely based on their impact on cellular redox-status. Instead, the lignans might affect more explicit biochemical pathways vital to chlamydial infection, and Schisandra lignans have previously been shown to affect many host-cellular pathways that are activated upon chlamydial replication. ${ }^{13,16,41,42}$ Schisandra lignans are promising antichlamydial natural products that can be used as tools to further understand the Chlamydia life cycle and starting points for discovery of novel antibacterial agents.

\section{ACKNOWLEDGEMENTS}

The work has been supported by Academy of Finland grant 252216 to LH, and Drug Discovery and Chemical Biology network of Biocenter Finland to PV. Laboratories for Chemical Biology Umeå thank the Swedish Research Council, the Swedish Governmental Agency for Innovation Systems, and the Knut \& Alice Wallenberg foundation for support. 
1 Vila-Corcoles, A., Ochoa-Gondar, O., Rodriguez-Blanco, T., Raga-Luria, X. \& Gomez-Bertomeu, F. Epidemiology of community-acquired pneumonia in older adults: a population-based study. Respir. Med. 103, 309-316 (2009).

2 Hahn, D. L. Chlamydia pneumoniae, asthma, and COPD: what is the evidence? Ann Allerg. Asthma Immunol. 83, 271-292 (1999).

3 Blasi, F. et al. Chlamydia pneumoniae and chronic bronchitis: association with severity and bacterial clearance following treatment. Thorax 57, 672-676 (2002).

4 Hasan, Z. N. Association of Chlamydia pneumoniae serology and ischemic stroke. South. Med. J. 104, 319-321 (2011).

5 Carter, J. D. \& Hudson, A. P. The evolving story of Chlamydia-induced reactive arthritis. Curr. Opin. Rheumatol. 22, 424-430 (2010).

6 Zhan, P. et al. Chlamydia pneumoniae infection and lung cancer risk: a meta-analysis. Eur. J. Cancer 47, 742-747 (2011).

7 Land, J. A., Van Bergen, J. E., Morre, S. A. \& Postma, M. J. Epidemiology of Chlamydia trachomatis infection in women and the cost-effectiveness of screening. Hum. Reprod. Update 16, 189-204 (2010)

8 Peipert, J. F. Genital chlamydial infections. N. Engl. J. Med. 349, 2424-2430 (2003).

9 Mariotti, S. P., Pascolini, D. \& Rose-Nussbaumer, J. Trachoma: global magnitude of preventable cause of blindness. Br. J. Ophthalmol. 93, 563-568 (2009).

10 Blasi, F. \& Tarsia, P. Value of short-course antimicrobial therapy in community-acquired pneumonia. Int. J. Antimicrob. Agents 26, S148-S155 (2005)

11 Hanski, L. \& Vuorela, P. M. Recent advances in technologies for developing drugs against Chlamydia pneumoniae. Expert. Opin. Drug. Discov. 9, 791-802 (2014).

12 Panossian, A. \& Wikman, G. Pharmacology of Schisandra chinensis Bail: an overview of Russian research and uses in medicine. J. Ethnopharmacol. 118, 183-212 (2008).

13 Guo, L. Y. et al. Anti-inflammatory effects of schisandrin isolated from the fruit of Schisandra chinensis Baill. Eur. J. Pharmacol. 591, 293-299 (2008).

14 Stacchiotti, A., Li Volti, G., Lavazza, A., Rezzani, R. \& Rodella, L. F. Schisandrin B stimulates a cytoprotective response in rat liver exposed to mercuric chloride. Food Chem. Toxicol. 47, 2834-2840 (2009).

15 Lee, T. H., Jung, C. H. \& Lee, D. Neuroprotective effects of Schisandrin B against transient focal cerebral ischemia in Sprague-Dawley rats. Food Chem. Toxicol. 50, 4239-4245 (2012)

16 Liu, K. \& Lesca, P. Pharmacological properties of dibenzocyclooctadiene derivatives isolated from Fructus Schizandrae chinensis III. Inhibitory effects on carbon tetrachloride-induced lipid peroxidation, metabolism and covalent binding of carbon tetrachloride to lipids. Chem. Biol. Interact. 41, 39-47 (1982).

17 Opletal, L., Sovová, H. \& Bártlová, M. Dibenzocyclooctadiene lignans of the genus Schisandra: importance, isolation and determination. J. Chromatogr. B Analyt. Technol. Biomed. Life Sci. 812, 357-371 (2004).

18 Zeng, K., Zhang, T., Fu, H., Liu, G. \& Wang, X. Schisandrin B exerts antineuroinflammatory activity by inhibiting the Toll-like receptor 4-dependent MyD88/ IKK/NF-KB signaling pathway in lipopolysaccharide-induced microglia. Eur. J. Pharmacol. 692, 29-37 (2012).

$19 \mathrm{Ip}, \mathrm{S}$. \& Ko, K. The crucial antioxidant action of schisandrin B in protecting against carbon tetrachloride hepatotoxicity in mice: a comparative study with butylated hydroxytoluene. Biochem. Pharmacol. 52, 1687-1693 (1996).

20 Chiu, P. Y., Leung, H. Y., Poon, M. K. T., Lee, S. S. T. \& Ko, K. M. Schisandrin B induced antioxidant response is partly mediated by cytochrome P-4502E1 catalyzed reaction in mouse liver. Mol. Cell. Biochem. 293, 87-92 (2006).

21 Zhang, B., Liu, Z. \& Hu, X. Inhibiting cancer metastasis via targeting NAPDH oxidase 4. Biochem. Pharmacol. 86, 253-266 (2013).

$22 \mathrm{Kim}, \mathrm{T}$. et al. Chlamydophila pneumoniae triggers release of CCL2O and vascular endothelial growth factor from human bronchial epithelial cells through enhanced intracellular oxidative stress and MAPK activation. J. Clin. Immunol. 29, 629-636 (2009).
23 Di Pietro, M., Filardo, S., De Santis, F. \& Sessa, R. Chlamydia pneumoniae infection in atherosclerotic lesion development through oxidative stress: a brief overview. Int. J. Mol. Sci. 14, 15105-15120 (2013).

24 Azenabor, A. A., Yang, S., Job, G. \& Adedokun, O. O. Elicitation of reactive oxygen species in Chlamydia pneumoniae-stimulated macrophages: $\mathrm{a} \mathrm{Ca}^{2}$-dependent process involving simultaneous activation of NADPH oxidase and cytochrome oxidase genes. Med. Microbiol. Immunol. (Berl.) 194, 91-103 (2005).

25 Azenabor, A. A. \& Chaudhry, A. U. Effective macrophage redox defense against Chlamydia pneumoniae depends on L-type $\mathrm{Ca}^{2}$ channel activation. Med. Microbiol. Immunol. (Berl) 192, 99-106 (2003).

26 Kuo, C. \& Grayston, J. T. A sensitive cell line, HL cells, for isolation and propagation of Chlamydia pneumoniae strain TWAR. J. Infect. Dis. 162, 755-758 (1990).

27 Alvesalo, J. et al. Inhibitory effect of dietary phenolic compounds on Chlamydia pneumoniae in cell cultures. Biochem. Pharmacol. 71, 735-741 (2006).

28 Caldwell, H. D., Kromhout, J. \& Schachter, J. Purification and partial characterization of the major outer membrane protein of Chlamydia trachomatis. Infect. Immun. 31, 1161-1176 (1981).

29 Sandberg, M., Määttänen, A., Peltonen, J., Vuorela, P. M. \& Fallarero, A. Automating a 96-well microtitre plate model for Staphylococcus aureus biofilms: an approach to screening of natural antimicrobial compounds. Int. J. Antimicrob. Agents 32, 233-240 (2008)

30 Karlsson, D. et al. The exploration of thienothiazines as selective butyrylcholinesterase inhibitors. Eur. J. Pharm. Sci. 47, 190-205 (2012).

31 Marwaha, S. et al. N-acylated derivatives of sulfamethoxazole and sulfafurazole inhibit intracellular growth of Chlamydia trachomatis. Antimicrob. Agents Chemother. 58, 2968-2971 (2014).

32 Ip, S., Ma, C., Che, C. \& Ko, K. Methylenedioxy group as determinant of schisandrin in enhancing hepatic mitochondrial glutathione in carbon tetrachloride-intoxicated mice. Biochem. Pharmacol. 54, 317-319 (1997).

33 Yim, T. K. \& Ko, K. M. Methylenedioxy group and cyclooctadiene ring as structural determinants of schisandrin in protecting against myocardial ischemia-reperfusion injury in rats. Biochem. Pharmacol. 57, 77-81 (1999).

34 Chiu, P. Y., Chen, N., Leong, P. K., Leung, H. Y. \& Ko, K. M. Schisandrin B elicits a glutathione antioxidant response and protects against apoptosis via the redox-sensitive ERK/Nrf2 pathway in H9c2 cells. Mol. Cell. Biochem. 350, 237-250 (2011).

35 Wolf, K., Fischer, E. \& Hackstadt, T. Ultrastructural analysis of developmental events in Chlamydia pneumoniae-infected cells. Infect. Immun. 68, 2379-2385 (2000).

$36 \mathrm{lp}$, S. P. et al. Schisandrin B protects against carbon tetrachloride toxicity by enhancing the mitochondrial glutathione redox status in mouse liver. Free Radic. Biol. Med. 21, 709-712 (1996).

37 Checker, R. et al. Schisandrin B exhibits anti-inflammatory activity through modulation of redox-sensitive transcription factors Nrf-2 and NF-kB. Free Radic. Biol. Med. 53 1421-1430 (2012).

38 Hancke, J., Burgos, R. \& Ahumada, F. Schisandra chinensis (Turcz.) Baill. Fitoterapia 70, 451-471 (1999).

39 Song, Q. et al. Lignans from the fruit of Schisandra sphenanthera, and their inhibition of HSV-2 and adenovirus. Phytochem. Lett. 6, 174-178 (2013).

$40 \mathrm{lp}$, S. P., Che, C. T. \& Ko, K. M. Structure-activity relationship of schisandrins in enhancing liver mitochondrial glutathione status in $\mathrm{CCl} 4$-poisoned mice. Zhongguo Yao Li Xue Bao 19, 313-316 (1998).

41 Yu, L., Liu, G., Sun, Y., Zhang, H. \& Zhang, H. Antioxidative effect of schisanhenol on human low density lipoprotein and its quantum chemical calculation. Acta Pharmacol. Sin. 25, 1038-1044 (2004).

42 Park, E. et al. Schisandrin B suppresses TGF $\beta 1$ signaling by inhibiting Smad2/3 and MAPK pathways. Biochem. Pharmacol. 83, 378-384 (2012).

Supplementary Information accompanies the paper on The Journal of Antibiotics website (http://www.nature.com/ja) 